\title{
Amelioration of caspase 3,7 activity by SPO I in human primary neurons exposed to HIV
}

\author{
This article was published in the following Dove Press journal: \\ Neurobehavioral HIV Medicine \\ 13 July 2010 \\ Number of times this article has been viewed
}

\begin{abstract}
Amol Shah'
Gursharan Chana',2

Ginger R Lucero'

Eliezer Masliah ${ }^{3}$

Cristian L Achim'

Laurent Lecanu ${ }^{4}$

Vassilios Papadopoulos ${ }^{4}$

Janet Greeson ${ }^{5}$

lan $\mathrm{P}$ Everall ${ }^{1,2}$

'Department of Psychiatry, HIV Neurobehavioral Research Center, University of California, San

Diego, School of Medicine, La Jolla, CA, 92093, USA; '2Department of Psychiatry, The University of Melbourne, Royal Melbourne Hospital, VIC, 3050, Australia; ${ }^{3}$ Department of Neurosciences, University of California, San Diego, School of Medicine, La Jolla, CA, 92093, USA;

${ }^{4}$ The Research Institute of the McGill University Health Centre and Department of Medicine, McGill University, Montreal QC, Canada; ${ }^{5}$ Samaritan Pharmaceuticals, Las Vegas, Nevada
\end{abstract}

Correspondence: lan Paul Everall Department of Psychiatry, The University of Melbourne, Royal Melbourne Hospital, VIC, 3050, Australia

Tel +6I-(0)3-8344-5955

Fax +6I-(0)3-9347-3457

Email ieverall@unimelb.edu.au

\begin{abstract}
Despite development of antiretroviral therapy (ARV) HIV associated neurocognitive disorder (HAND) continues to be a significant cause of morbidity. As ARVs are not entirely effective against HAND, there is significant motivation to discover novel therapies that are targeted towards the treatment of HAND. A novel formulation of procaine hydrochloride, SP01, is one potential molecule which has shown promise. Phase I clinical trials have found SP01 treatment to significantly increase CD4 cell counts and quality of life parameters in HIV infected patients. To assess SP01's potential use for HAND we utilized an in vitro human primary neuron culture model and conducted co-exposure experiments with gp120 ([BaL] 300 pg/mL) or HIV (BaL) $500 \mathrm{pg} / \mathrm{mL})$ with or without SP01 $(10 \mu \mathrm{M})$ and measured the activity of the pro-apoptotic caspases $3 / 7$ using a quantitative luminescence assay. Both gp 120 and HIV resulted in a significant increase in caspase $3 / 7$ activity from controls $(P<0.05)$. This effect was ameliorated with coexposure with SP01 $(P<0.05)$. The reduction in activity of caspases 3 and 7 , which are well known to be cellular triggers for apoptosis, signifies that SP01 is likely to have neuroprotective effects against HIV induced neurodegeneration via inhibition of pro-apoptotic cascades. Given its success in clinical trials and in vitro experiments, SP01 seems to be a legitimate contender in the fight against HAND.
\end{abstract}

Keywords: HIV, SP01, neurotoxicity, caspase, HAND, neuroprotection

\section{Introduction}

Human immunodeficiency virus (HIV) is a global pandemic with numbers increasing from eight million in 1990 to 33 million in $2007 .{ }^{1}$ As a neurotropic retrovirus, HIV targets the brain resulting in inflammatory changes such as HIV encephalitis (HIVE) and HIV leukoencephalopathy along with neuronal and synapto-dendritic marker loss. ${ }^{2,3}$ These changes, observed in post-mortem brain tissue, have been shown to correlate with the clinical severity of HIV associated neurocognitive disorder (HAND) in corresponding patients prior to death. ${ }^{2-4}$ Unfortunately, HAND continues to be a persistent problem despite development of antiretroviral therapy ${ }^{5}$ (ARV); with prevalence of mild to moderate degrees of HAND, approximately $55 \%$, before and after the introduction of ARV. ${ }^{6}$ As ARVs do not appear to be totally effective in treating HAND there is an impetus to develop brain targeted adjunctive therapies that can prevent or ameliorate neurocognitive impairments in the longer surviving HIV infected population. In relation to the pathophysiology of HAND, elevated levels of cortisol have been seen in individuals with advanced HIV with hypercortisolemic states also being seen in patients with mood disorders where cognitive deficits are also apparent. ${ }^{7,8}$ As anti-cortisol drugs have being shown submit your manuscript | www.dovepress.com

Dovepress

8711 
to reduce infectivity and replication of HIV in vitro they represent a potentially novel therapeutic target for treating HAND. ${ }^{9}$ Due to cortisol being synthesized from cholesterol, cholesterol represents an additional target to reduce hypercortisolemic states. Procaine hydrochloride (SP01) has been shown in vitro to reduce levels of 3-hydroxy-3methylglutaryl-coenzyme A (HMG-CoA) reductase, ${ }^{10}$ a key enzyme that converts $\mathrm{HMG}-\mathrm{CoA}$ to mevalonate during the synthesis of cholesterol. Cholesterol is also found within membrane lipid rafts that are important in modulating the expression of membrane receptors such as CCR5 which are essential for HIV cell fusion.

SP01A (an oral formulation of procaine hydrochloride by Samaritan Pharmaceuticals, Las Vegas, NV, USA) has also has been reported to reduce membrane cholesterol and plasma HIV viral load in vitro. ${ }^{11}$ In addition, SP01 has been shown to protect rat neuronal cells against glutamate- and $\beta$-amyloid-induced toxicity. ${ }^{12}$ Due to its lipophilic nature, SP01 has good brain penetrance, making it a worthy candidate as a potential neuroprotective agent adjunct to existing therapies. In a study of $30 \mathrm{HIV}$-infected patients from Brazil, France and the USA, CD4 cell counts were increased by 68 cells/month in those that received SP01 alone and 44 cells/month in those that received SP01 and zidovudine versus controls. Additionally, most patients showed continued improvement in their quality of life parameters. ${ }^{13}$ Given the relative success of SP01 in phase I clinical trials, we decided to further examine its neuroprotective potential in human primary neurons exposed to HIV (BaL). Cells we have previously shown to undergo neurodegenerative effects in response to HIV (BaL). ${ }^{14}$ In addition we exposed neurons to gp120 (BaL), a key envelop glycoprotein of HIV which we have shown previously to be neurotoxic in human primary human neurons. ${ }^{15}$ Our overall aim in this proposal was to provide further evidence for the use of orally administered SP01 to be further developed for the treatment of HAND.

\section{Materials and methods}

\section{Establishment of primary human neuronal cultures}

Human primary neuronal cultures were prepared as previously described by Trillo-Pazos and colleagues, ${ }^{16}$ from human fetal brain tissue, 14-18 weeks gestation, collected from consenting patients undergoing terminations without any clinical identifiers (Advanced Bioscience Resources, CA, USA). Cultures were maintained for a period of 30 days prior to experimentation, corresponding to the beginning of
NMDA receptor expression in these cultures and as such representative of a functionally mature phenotype. ${ }^{17}$

\section{Assessment of neurotoxicity of SPO I}

After four weeks in culture, neurons were exposed to a SP01 in a concentration range of $100 \mathrm{nM}, 1 \mu \mathrm{M}, 10 \mu \mathrm{M}$, and $1 \mathrm{mM}$; concentrations at which procaine has been shown not to negatively affect neuronal cell viability. ${ }^{18}$ Time points were taken at 6 hours, 24 hours, and 72 hours, with lactate dehydrogenase (LDH) assays taken (Cytotox 96 kit, Promega, Madison, WI, USA) as a measure of neuronal toxicity.

\section{Exposure of neurons to gP 120}

Following a four week maturation period as described above, human primary neurons were exposed to the following conditions: untreated (1:1000 DMSO as a vehicle control), gp120 (300 pg/mL), SP01 [10 $\mathrm{MM}$ ], gp120 + SP01 $[10 \mu \mathrm{M}]$. The neurons were incubated at $37^{\circ} \mathrm{C}$ for 12 hours after which they were assayed for the apoptosis effector proteins caspase 3 and 7 using the Caspase-Glo 3/7 assay kit (Promega, USA).

\section{Growth of HIV (BaL) in macrophages}

Peripheral blood mononuclear cells (PBMCs) were isolated from the buffy coats procured from the San Diego Blood Bank using the Histopaque reagent and protocol (Sigma, St Louis, MO, USA). PBMCs were cultured in media to promote growth of macrophages (Iscove's modified Dulbecco's media [IMDM] $+10 \%$ heat inactivated human serum $+1 \%$ penicillin and streptomycin). HIV-1 (BaL) viral stock was obtained from the NIH AIDS Research and Reference Reagent Program (Cat \#510, Lot \#060493) and used to infect the macrophages at a final viral core protein (p24) concentration of $1000 \mathrm{pg} / \mathrm{mL}$ for 12 hours, followed by a washout with phosphate buffered saline. p24 values were then monitored every three days. After four weeks, the p24 values had peaked to $2000 \mathrm{pg} / \mathrm{mL}$ and the infected macrophage conditioned media (MCM) was collected and stored at $-20^{\circ} \mathrm{C}$.

\section{Exposure to neurons to HIV (BaL)}

Following four weeks of culturing, neurons were exposed to the following conditions: uninfected (20\% uninfected macrophage media), 25\% BaL-MCM (500 pg/mL), $10 \mu \mathrm{M}$ $\mathrm{SP} 01+25 \% \mathrm{BaL}-\mathrm{MCM}$. The neurons were incubated at $37^{\circ} \mathrm{C}$ for 12 hours after which they were assayed for apoptosis effector proteins caspase 3 and 7 using the Caspase-Glo 3/7 assay kit (Promega, USA). 


\section{Results}

\section{Assessment of neurotoxicity of SPOI}

No differences in LDH were observed between the control, untreated neurons, and neurons treated with SP01 at all concentrations $(P>0.10)$.

\section{Exposure of neurons to gp 120}

Measurement of neuronal cell caspase 3 and 7 activity levels indicated that SP01 is neuroprotective against gp120 $(300 \mathrm{pg} / \mathrm{mL})$. As can be seen in Figure 1 there were statistically significant differences in the exposure groups versus controls (ANOVA, F = 29.45, $\mathrm{df}=3, P=0.003$ ). Post-hoc analysis of the groups revealed that gp 120 on its own resulted in a 7\% increase in caspase 3 and 7 activity $(P=0.046$ while co-exposure to gp120 infected macrophage media in the presence of SP01 $[10 \mu \mathrm{M}]$ reduced the caspase 3 and 7 activity by $13 \%$ from gp120 alone $(P=0.02)$. These observations demonstrate that SP01, at a non-toxic dose, was capable of preventing gp120 associated apoptosis.

\section{Exposure to neurons to HIV (BaL)}

Determination of neuronal cell caspase 3 and 7 activity levels indicated that SP01 is also neuroprotective against HIV $(500 \mathrm{pg} / \mathrm{mL})$. As can be seen in Figure 2 there were statistically significant differences in the exposure groups versus controls $(\mathrm{F}=65.2, \mathrm{df}=2, P<0.0001)$. Exposure of the primary human

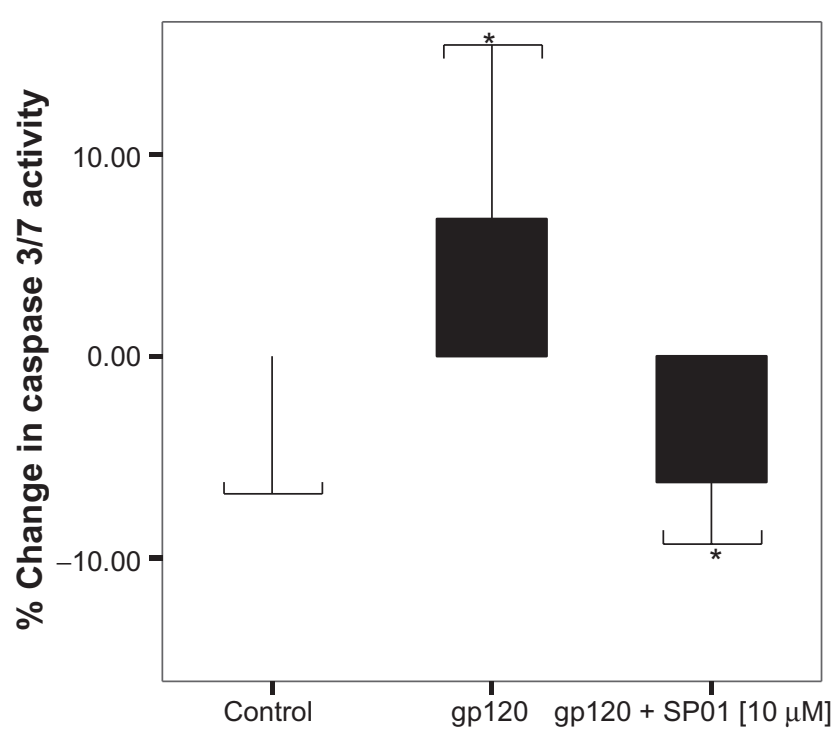

Figure I Caspase 3 and 7 activities in human primary neurons exposed to gP I 20 and SPOI. Notes: Human primary neurons were incubated in the presence and absence of gPI $20(\mathrm{BaL})$ at $300 \mathrm{pg} / \mathrm{mL}$ with and without SPOI [10 $\mu \mathrm{M}$ ]. Exposure of neurons to $\mathrm{gPl} 20$ at $300 \mathrm{pg} / \mathrm{mL}$ resulted in a significant increase in caspase 3,7 activity $(P=0.046)$ that was ameliorated by co-exposure with $[10 \mu \mathrm{M}] .(P=0.02)$. Units on $y$-axis represent luminescence as a direct measure of caspase 3 and 7 activity, data points represent means and standard deviations for measures. neurons to media from HIV infected macrophages resulted in a $40 \%$ increase in caspase 3 and 7 activation $(P<0.0001)$ while co-exposure to HIV infected macrophage media in the presence of SP01 $[10 \mu \mathrm{M}]$ reduced the caspase 3 and 7 activity by $11 \%$ from $\mathrm{HIV}(\mathrm{BaL})$ alone $(P=0.026)$.

\section{Discussion}

In this study we observed that SP01 is neuroprotective against gp120 (BaL) and HIV (Bal) induced apoptosis in primary human neurons. Previous studies have shown that HIV causes neurodegeneration via activation of caspase-dependent apoptotic pathways ${ }^{19}$ and that caspase 3 was found to be overly expressed in HIV-1 encephalitis. ${ }^{20}$ In our study, values of caspase 3 and 7 were specifically measured as they are effector caspases known to trigger key cellular apoptotic processes. In addition, LDH assays for neuronal toxicity confirmed that at the tested concentrations, SP01 was not toxic. While the exact mechanism of SP01 activity is uncertain, it is hypothesized that SP01 may modify the cholesterol content of cell membranes, thereby inhibiting HIV fusion. It has been shown that SP01 reduces stress induced 3-hydroxy3-methyl-glutaryl-CoA (HMG-CoA) reductase mRNA expression, leading to a reduction in cholesterol synthesis. ${ }^{10}$ Evidence shows that cholesterol-enriched rafts containing CD4, CXCR4, and CCR5 receptors are essential for HIV to bind and fuse with the target cell membranes. ${ }^{21,22}$ Therefore, inhibitors of cholesterol synthesis such as SP01 may be able to prevent or inhibit cell fusion by HIV. It is also possible that SP01 is capable of modulating pathological dysregulation of circulating elevated cortisol in HIV positive subjects. Elevated cortisol levels have been found in HIV infection and are thought to play a role in the pathophysiology of AIDS, including modulation of cell-mediated immunity. ${ }^{23}$ Studies into the mechanism behind SP01 modulation of cholesterol synthesis will be critical for identifying its role in HIV induced cortisol dysregulation and neuropathogenesis.

In conclusion, the findings from this investigation provide evidence for SP01 having neuroprotective effects against HIV induced neurodegeneration, via inhibition of pro-apoptotic cascades. SP01's ability to prevent HIV mediated neurotoxicity and neurodegenerative effects have implications in potentially alleviating symptoms of HAND. Furthermore, as we did not observe neurotoxicity with the concentrations of SP01 used in this investigation, and given that SP01 is well tolerated in patients, supports its potential as a therapeutic agent in treating HAND. While this investigation assessed markers of pro-apoptotic events via assessment of caspase 3 and 7, we did not look at other potential markers of apoptosis such as the 


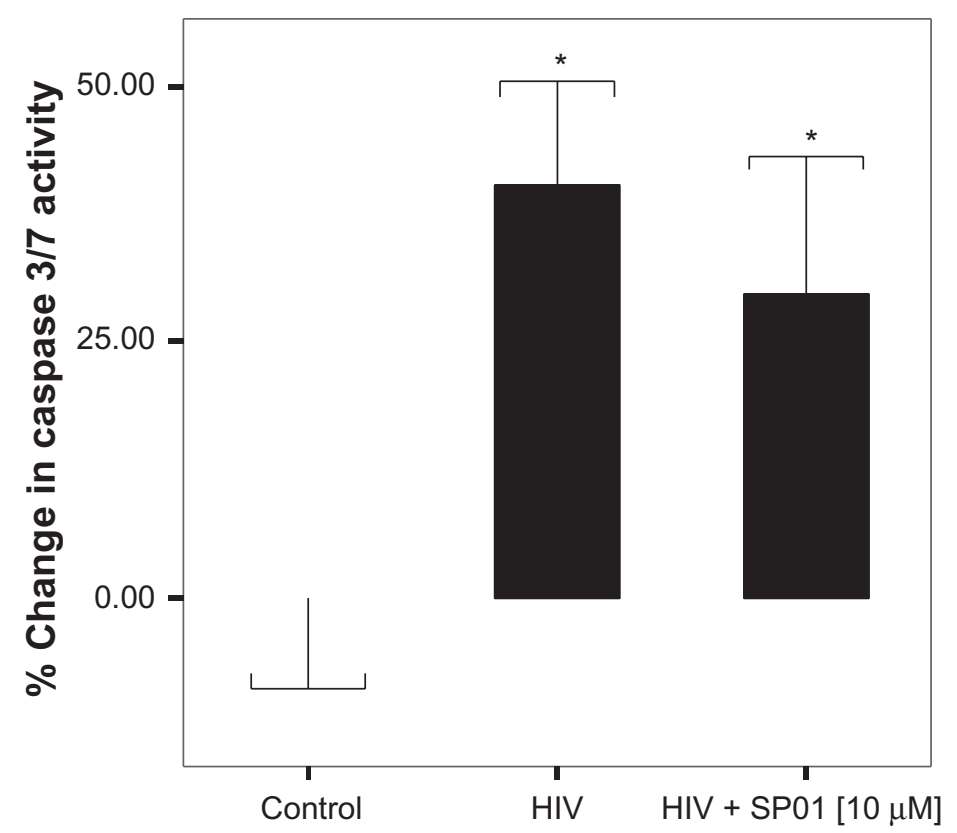

Figure 2 Caspase 3 and 7 activity in human primary neurons exposed to HIV BaL and SPOI.

Notes: Human primary neurons were incubated in the presence and absence of gP I 20 (BaL) at $300 \mathrm{pg} / \mathrm{mL}$ with and without SPOI [I0 $\mu \mathrm{M}$ ]. Exposure of neurons to HIV (BaL) $[500 \mathrm{pg} / \mathrm{mL}]$ resulted in a significant increase in caspase 3 and 7 activity $(P<0.000 \mathrm{I})$ which was ameliorated with co-exposure to SPOI [I0 $\mu M](P=0.026)$. Units on $y$-axis represent luminescence as a direct measure of caspase 3 and 7 activity, data points represent means and standard deviations for measures.

Abbreviation: HIV, human immunodeficiency virus.

ratio of anti-apoptotic factors such as $B C L X$ and $B C L 2$ to proapoptotic markers such as $B A D$ or $B A X$. In addition, while we evidenced the neuroprotective effects of SP01 in ameliorating caspase induction caused by HIV we were not able to ascertain the exact mechanism of this effect. Therefore, our future work will focus on assessing additional markers of apoptosis as well as investigating the effects of SP01 on CD4, CXCR4 and CCR5 receptor expression on the cell membrane; as well as assessing the direct downstream effects of SP01 and correlating with further markers of neurodegeneration, such as synaptic and dendritic markers. We will also carry out this work over a longer course of HIV infection and treatment with SP01 in order to assess the SP01 sustained neuroprotection.

\section{Acknowledgments}

This work was supported by an NIH R41 MH79728 small business technology transfer (STTR) between University of California, San Diego and Samaritan Pharmaceuticals, Las Vegas, Nevada. This work was also supported by NIH Grant MH62962.

\section{References}

1. 2008 Report on the global AIDS epidemic. Joint United Nations Programme on HIV/AIDS;UNAIDS 2008.

2. Everall IP, Heaton RK, Marcotte TD, et al. Cortical synaptic density is reduced in mild to moderate human immunodeficiency virus neurocognitive disorder. HNRC Group. HIV Neurobehavioral Research Center. Brain Pathol. 1999;9:209-217.
3. Masliah E, Heaton RK, Marcotte TD, et al. Dendritic injury is a pathological substrate for Human Immunodeficiency Virus-related cognitive disorders. Ann Neurol. 1997;42:963-972.

4. Cherner M, Masliah E, Ellis RJ, et al. Neurocognitive dysfunction predicts postmortem findings of HIV encephalitis. Neurology. 2002;59: $1563-1567$.

5. Clifford DB. HIV-associated neurocognitive disease continues in the antiretroviral era. Top HIV Med. 2008;16:94-98.

6. Tozzi V, Balestra P, Bellagamba R, et al. Persistence of neuropsychologic deficits despite long-term highly active antiretroviral therapy in patients with HIV-related neurocognitive impairment: prevalence and risk factors. J Acquir Immune Defic Syndr. 2007;45:174-182.

7. Leserman J. Role of depression, stress, and trauma in HIV disease progression. Psychosom Med. 2008;70:539-545.

8. Spijker AT, van Rossum EF. Glucocorticoid receptor polymorphisms in major depression. Focus on glucocorticoid sensitivity and neurocognitive functioning. Ann NY Acad Sci. 2009;1179:199-215.

9. Brown SJ, Fareed G, Codina S, et al. Procaine hydrochloride: in vitro antiretroviral properties and reduction of viral load in HAART-resistant patients. In: 2004 International Meeting of the Institute of Human Virology, Oct 31-Nov 4: Baltimore, MD, USA; 2004.

10. Xu J, Lecanu L, Han Z, Yao Z, Greeson J, Papadopoulos V. Inhibition of adrenal cortical steroid formation by procaine is mediated by reduction of the cAMP-induced 3-hydroxy-3-methylglutaryl-coenzyme A reductase messenger ribonucleic acid levels. J Pharmacol Exp Ther. 2003;307:1148-1157.

11. Brown SJ, Lecanu L, Fareed G, et al. Procaine oral stabilised-Samaritan pharmaceuticals: SP-01, SP001. Drugs R D. 2004;5:108-109.

12. Lecanu L, Wenguo Y, Xu J, Greeson J, Papadopoulos V. Local anesthetic procaine protects rat pheochromocytoma PC12 cells against beta-amyloid-induced neurotoxicity. Pharmacology. 2005;74:65-78.

13. de Moraes HB SA, Bensabat S. A pilot clinical study on $60 \mathrm{HIV}+$ and AIDs patients, treated with Anticort ${ }^{\circledR}$. In: International Conference on AIDS 2 Las Vegas, NV, USA; 1996. p. 91. 
14. Nguyen TB, Lucero GR, Chana G, et al. Glycogen synthase kinase-3beta (GSK-3beta) inhibitors AR-A014418 and B6B3O prevent human immunodeficiency virus-mediated neurotoxicity in primary human neurons. J Neurovirol. 2009;15(5-6):434-438.

15. Everall IP, Trillo-Pazos G, Bell C, Mallory M, Sanders V, Masliah E. Amelioration of neurotoxic effects of HIV envelope protein gp120 by fibroblast growth factor: a strategy for neuroprotection. J Neuropathol Exp Neurol. 2001;60:293-301.

16. Trillo-Pazos G, Kandanearatchi A, Eyeson J, King D, Vyakarnam A, Everall I. Infection of stationary human brain aggregates with HIV-1 sf162 and IIIB results in transient neuronal damage and neurotoxicity. Neuropathol Appl Neurobiol. 2004;30:136-147.

17. Mattson MP, Wang H, Michaelis EK. Developmental expression, compartmentalization, and possible role in excitotoxicity of a putative NMDA receptor protein in cultured hippocampal neurons. Brain Res. 1991;565:94-108.

18. Perez-Castro R, Patel S, Garavito-Aguilar ZV, et al. Cytotoxicity of local anesthetics in human neuronal cells. Anesth Analg. 2009;108: 997-1007.
19. Acquas E, Bachis A, Nosheny RL, Cernak I, Mocchetti I. Human immunodeficiency virus type 1 protein gp120 causes neuronal cell death in the rat brain by activating caspases. Neurotox Res. 2004;5:605-615.

20. James HJ, Sharer LR, Zhang Q, et al. Expression of caspase-3 in brains from paediatric patients with HIV-1 encephalitis. Neuropathol Appl Neurobiol. 1999;25:380-386.

21. Kozak SL, Heard JM, Kabat D. Segregation of CD4 and CXCR4 into distinct lipid microdomains in T lymphocytes suggests a mechanism for membrane destabilization by human immunodeficiency virus. J Virol. 2002;76:1802-1815.

22. Manes S, del Real G, Lacalle RA, et al. Membrane raft microdomains mediate lateral assemblies required for HIV-1 infection. EMBO Rep. 2000;1:190-196.

23. Kumar M, Kumar AM, Waldrop D, Antoni MH, Eisdorfer C. HIV-1 infection and its impact on the HPA axis, cytokines, and cognition. Stress. 2003;6:167-172.
Neurobehavioral HIV Medicine

\section{Publish your work in this journal}

Neurobehavioral HIV Medicine is an international, peer-reviewed, open access journal focusing on advances in research in HIV/ AIDS, with specific reference to the neurological, psychiatric and behavioral consequences of the disease, concomitant infections and specific antiretroviral therapy. The manuscript

\section{Dovepress}

management system is completely online and includes a very quick and fair peer-review system, which is all easy to use. Visit http://www.dovepress.com/testimonials.php to read real quotes from published authors.

Submit your manuscript here: http://www.dovepress.com/journal-of-neurobehavioral-hiv-medicine-journal 